\title{
CENTRO DE ARTES Y OFICIOS MOMPOX, BOLÍVAR
}

Víctor Andrés Tafurt Báez*

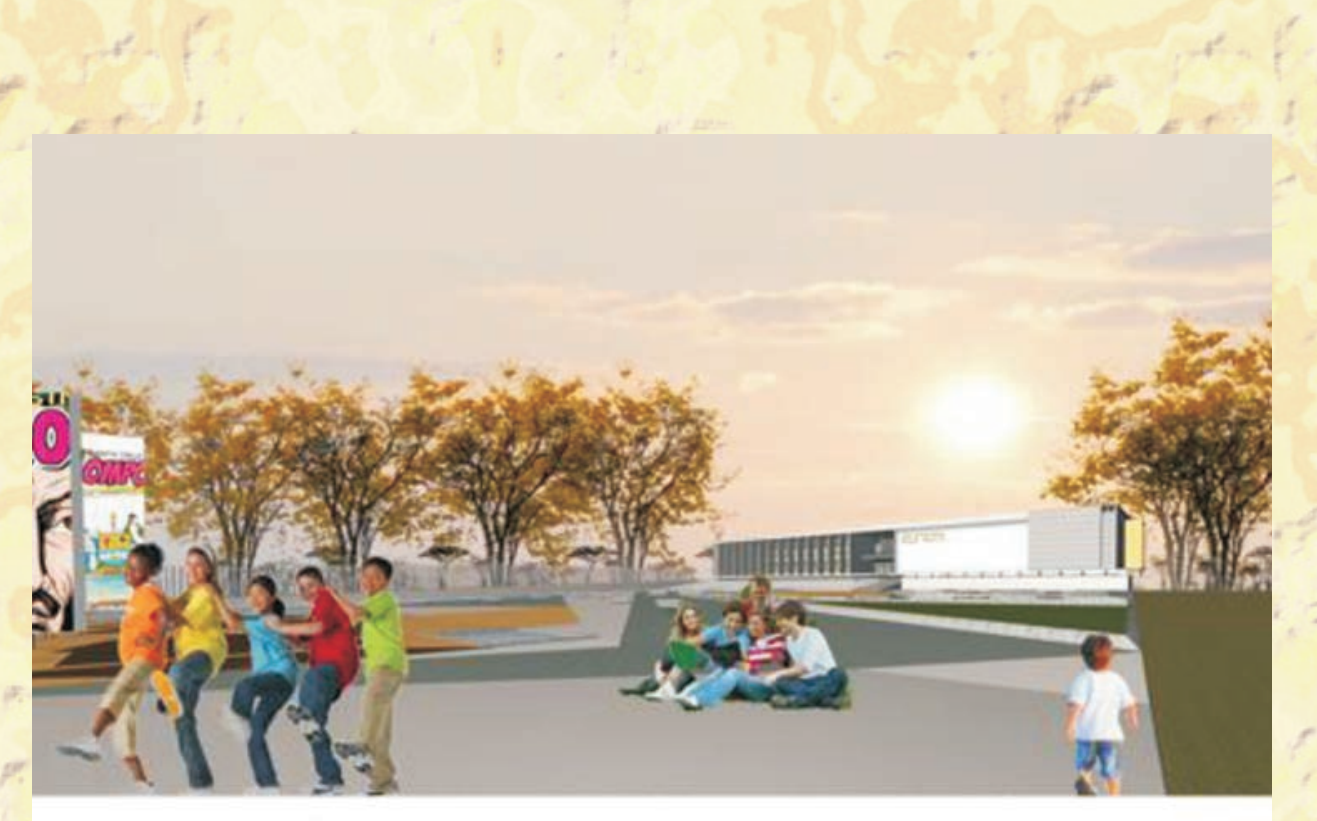

Imagen A. Propuesta de diseño de un Centro de Artes y Oficios en Mompox Fuente: Elaboración del autor

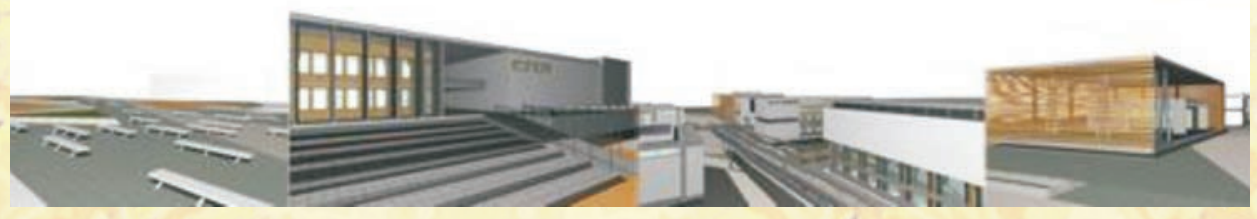

\section{RESUMEN}

La propuesta tuvo por objeto el desarrollo de un Centro de Bellas Artes en Mompox, al tiempo que la oferta de un conjunto de alternativas de alto nivel, dirigidas a aquellas personas con un interés por las artes y la cultura en general. La idea se desarrolló con el ánimo de contribuir con el destacado movimiento cultural y artístico de la región, de allí la necesidad de diseñar un espacio a través del cual se promoviera la educación y la cultura, con áreas para el desarrollo de actividades lúdicas dirigidas a la población infantil y juvenil, la capacitación de la comunidad y la formación artística. En el proyecto se evidencia la inclusión de las disciplinas tradicionales y los nuevos lenguajes y técnicas contemporáneas como estrategias para fortalecer y proseguir la tarea cultural, componente esencial para la convivencia social y el desarrollo humano; en síntesis, aportar a la comunidad en el ejercicio académico, el diseño de un escenario en el cual se estimulara de forma permanente el desarrollo y la capacitación de las artes y la cultura en Mompox, Bolívar.

\section{PALABRAS CLAVE}

Proyecto arquitectónico, Centro de artes y oficios, Mompox, Bolívar, Patrimonio, Sociedad. 
ARTS AND CRAFTS CENTER, MOMPOX, BOLIVAR Víctor Andrés Tafurt Báez*

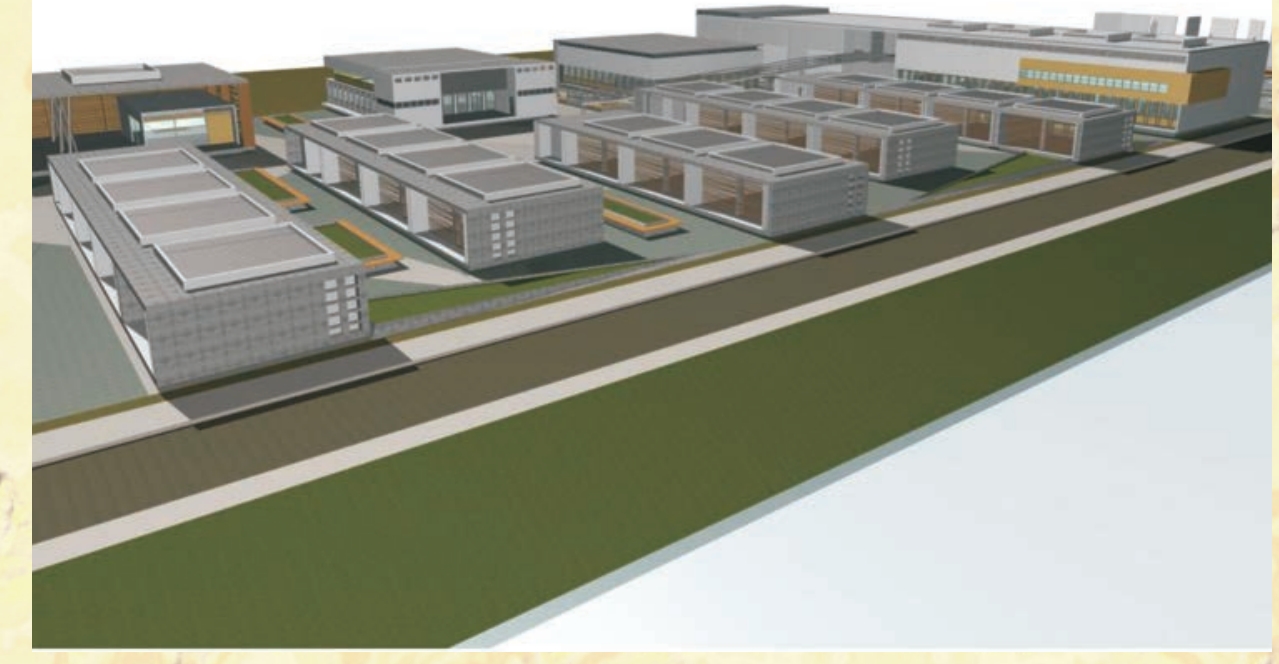

\section{ABSTRACT}

The proposal aimed to develop an Arts and Crafts center in Mompox and also a set of high level alternatives, thinking about people mainly interested in arts and culture. The idea was developed observing the importance of contributing to the cultural and artistic movement in the region, hence the need to design a space to promote education and culture, through areas for recreational activities of children and youth as well for community training and artistic education. The project highlights the inclusion of traditional disciplines, the new languages and contemporary techniques as a strategy to strengthen the task of continuing culture, an essential component for social cohesion and human development. To summarize, provide the community, throughout an academic exercise, the project of a "scenario" that will permanently stimulate the development and training of arts and culture in Mompox, Bolívar.

\section{KEY WORDS}

architectonic project, arts and crafts center, Mompox, Bolivar, Heritage, Society.
Este artículo es producto de la investigación realizada para optar por el título de arquitecto en la Facultad de Arquitectura de la Universidad Santo Tomás, seccional Bucaramanga, la cual fue calificada como meritoria.

* El proyecto fue dirigido por el arquitecto Hernando Ladino Barriga. Actuaron en calidad de jurados los arquitectos Liliana Rueda Cáceres y Carlos Fernando Jaimes Ochoa.

Imagen B . Perspectiva del Centro de Artes y Oficios en Mompox. Fuente: Elaboración del autor 


\section{FICHA TÉCNICA DE LA PROPUESTA}

Composición Arquitectónica. La propuesta se desarrolló a partir de un eje peatonal tipo claustro, que enlaza diferentes espacios destinados a la ejecución de las artes y oficios; para ello, se diseña una biblioteca, áreas de esparcimiento, cafetería y salón comunal, al igual que salas de exposiciones destinadas a cultivar el espíritu lúdico y recreativo de la población. Este gran núcleo se enlaza con el resto del municipio a través de la plaza Cívica de Mompox. Los ejes planteados en el desarrollo de la propuesta se encuentran paralelos a los ejes ambientales, gracias al análisis arquitectónico y urbano realizado en el municipio. El desarrollo del espacio público y de los espacios arquitectónicos, tomó como punto de referencia el oficio de la filigrana como elemento identificador de la población, evidente en la forma y concepto que se le dio al tratamiento de los espacios: atrio, patios y claustro.

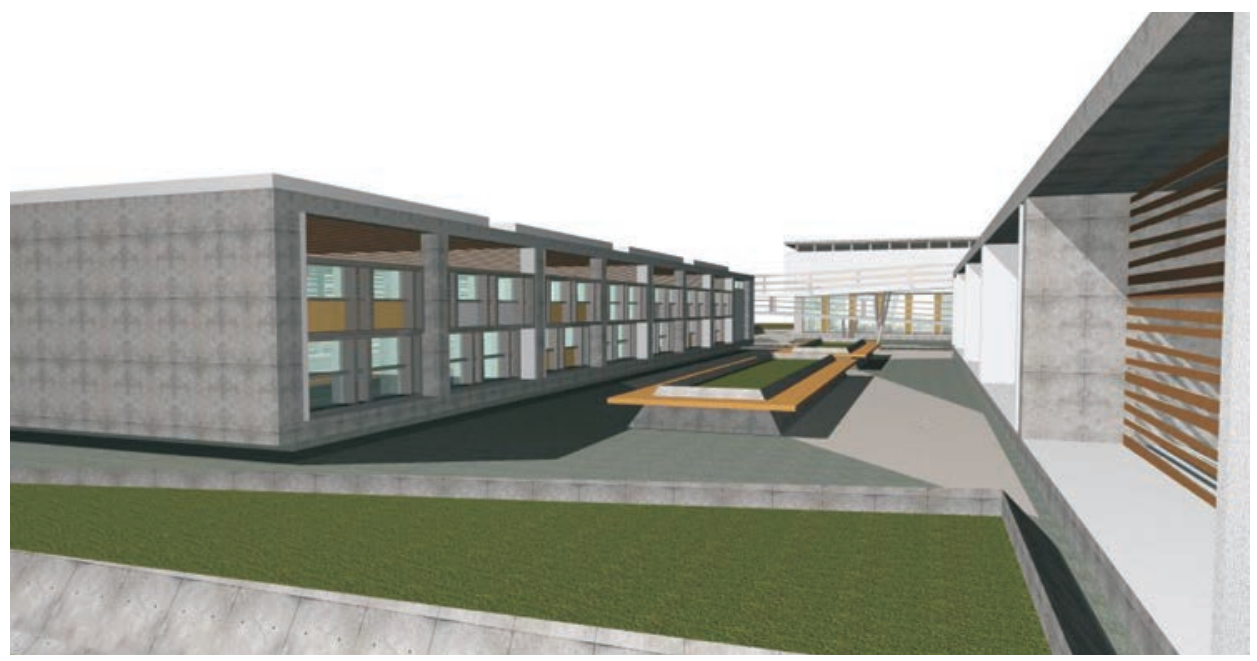

Propuesta de ocupación del proyecto. Se buscaba que la ocupación respondiera a un carácter público que permitiera el estímulo y la conexión de la comunidad momposina con su patrimonio histórico y cultural.

Zonificación. La zonificación del proyecto se desarrolló a partir de ejes paralelos a los ejes ambientales de la Albarrada y el Brazo del Río Magdalena, y de acuerdo a las condicionantes del lote (forma, soleamiento y vientos); esta geometrización generó por tanto un enlace con la estructura urbana existente (Albarrada, plazas y parques)

El espacio núcleo del proyecto. El proyecto estableció como principio el desarrollo apropiado del espacio público en su totalidad, se diseñaron para ello diferentes espacios destinados al peatón (Plaza Cívica Mompox) y patios entre los espacios arquitectónicos, para permitir un alto grado de accesibilidad. La Plaza Cívica de Mompox se convierte por tanto en el módulo de relación de la población con el Centro de Artes y Oficios, como enlace entre la arquitectura del lugar y la arquitectura proyectada basada en el neoplasticismo y como espacio cultural de la región.

\section{INTRODUCCIÓN}

La importancia de la historia, la sociedad y la cultura y en ella el arte en la formación del ser humano, permite generar dispositivos éticos y morales que conllevan a la formación de una sociedad que valora la importancia de la educación como una necesidad primaria y 


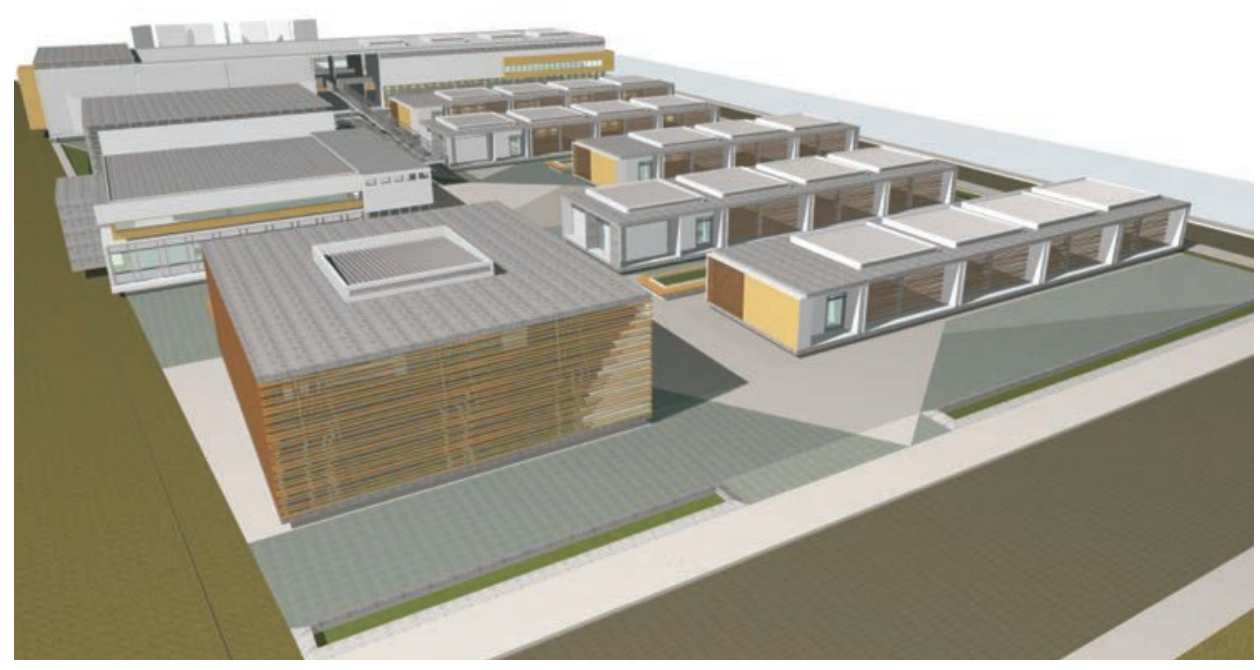

Imagen 2. Perspectiva general del Centro de Artes y Oficios Fuente: Elaboración del autor

constituyente: la posibilidad de escapar del proceso de deshumanización que se vive en la actualidad, una realidad de la cual no es posible hacerlo más que por medio de los valores que en la sociedad se promuevan. Por consiguiente, el desarrollo de un proyecto educativo, en este caso un Centro de Artes y Oficios en Mompox, se torna elemento moderador de la comunidad en la medida en que promueve relaciones de igualdad y encuentro con las artes. Sin embargo, no debemos olvidar que el desarrollo de una sociedad democrática está relacionado con otros factores primordiales como la posibilidad de acceder a la educación, el trabajo y la cultura, elementos que se han querido poner de presente en el desarrollo de esta propuesta arquitectónica.

Cabe resaltar que para la sociedad se torna importante poder contar con un espacio que le permita identificarse como comunidad $y$, a través del cual, puedan expresar sus ideas de manera creativa; de esa relación vital entre arte y sociedad surge la propuesta de un elemento arquitectónico cuya característica es la apropiada disposición de un sistema espacial y el fomento de las actividades artísticas y culturales de la región, elemento creado con una directriz precisa: generar un espacio donde prospere la creatividad, se motive la enseñanza y el aprendizaje y se valore la cultura por medio de procesos de participación ciudadana.

\section{PLANTEAMIENTO DEL PROBLEMA}

El proyecto pretende diseñar espacios en los cuales sea posible incentivar programas educativos que faciliten el proceso cognitivo y práctico de los niños y jóvenes en relación con su patrimonio artístico y cultural, que genere oportunidades de ingreso a la educación superior, rescate el patrimonio histórico de la región, garantice el desarrollo de espacios a través de los cuales se acceda al conocimiento de las artes y oficios. Es importante tener en cuenta la problemática a nivel educativo, aspecto de gran importancia, ya que es un proceso multidireccional mediante el cual se construyen y transmiten conocimientos, valores, costumbres y formas de actuar. En Mompox el 70\% de los bachilleres no tienen oportunidades laborares, a pesar de que un $9.4 \%$ corresponde a profesionales técnicos y tecnólogos. La deficiencia en el nivel educativo se evidencia en la baja tasa de escolaridad entre los estudiantes con edades que oscilan entre los 3 a los 17 años, la deserción escolar en todos los niveles es una constante que afecta el desarrollo educativo, ya que la mayoría de los niños y jóvenes trabajan, obteniendo ingresos mínimos e impidiendo su acceso a la educación primaria, básica, media y universitaria. 
Plano I. Localización Fuente: Plan Especial de Manejo y Protección del Centro Histórico de Santa Cruz de Mompox, Convenio No. 1403 de 2007. Agencia Española de Cooperación para el desarrollo, Ministerio de Cultura, Alcaldía de Mompox, Gobernación de Bolívar. Sandra Karime Zabala Corredor [Consultora] Licitación Pública No. 004 de 2007. Contrato 002 de 2007. Alcaldía Mompox.

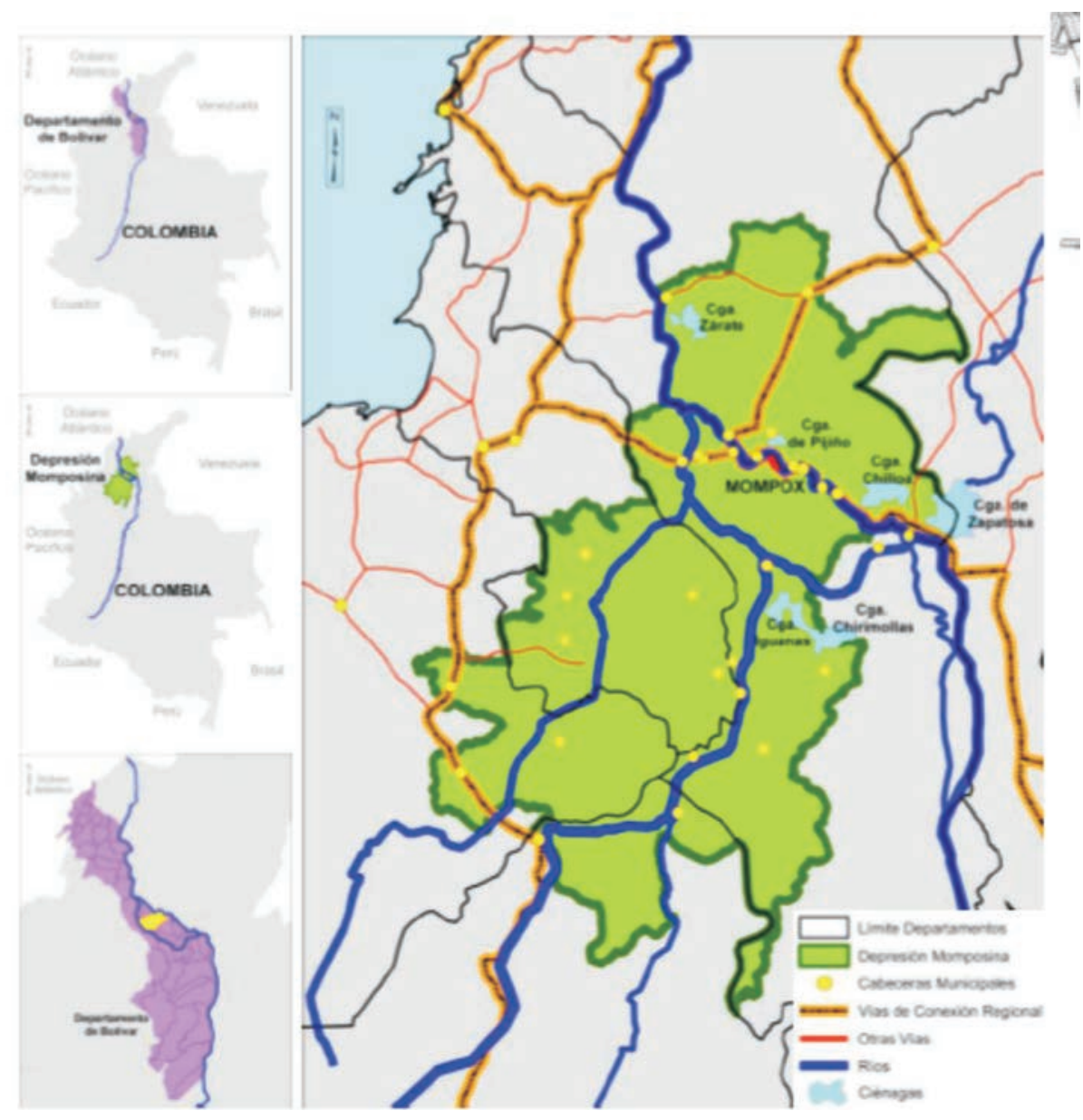

Imagen 3. Planta arquitectónica. Fuente: Elaboración del autor

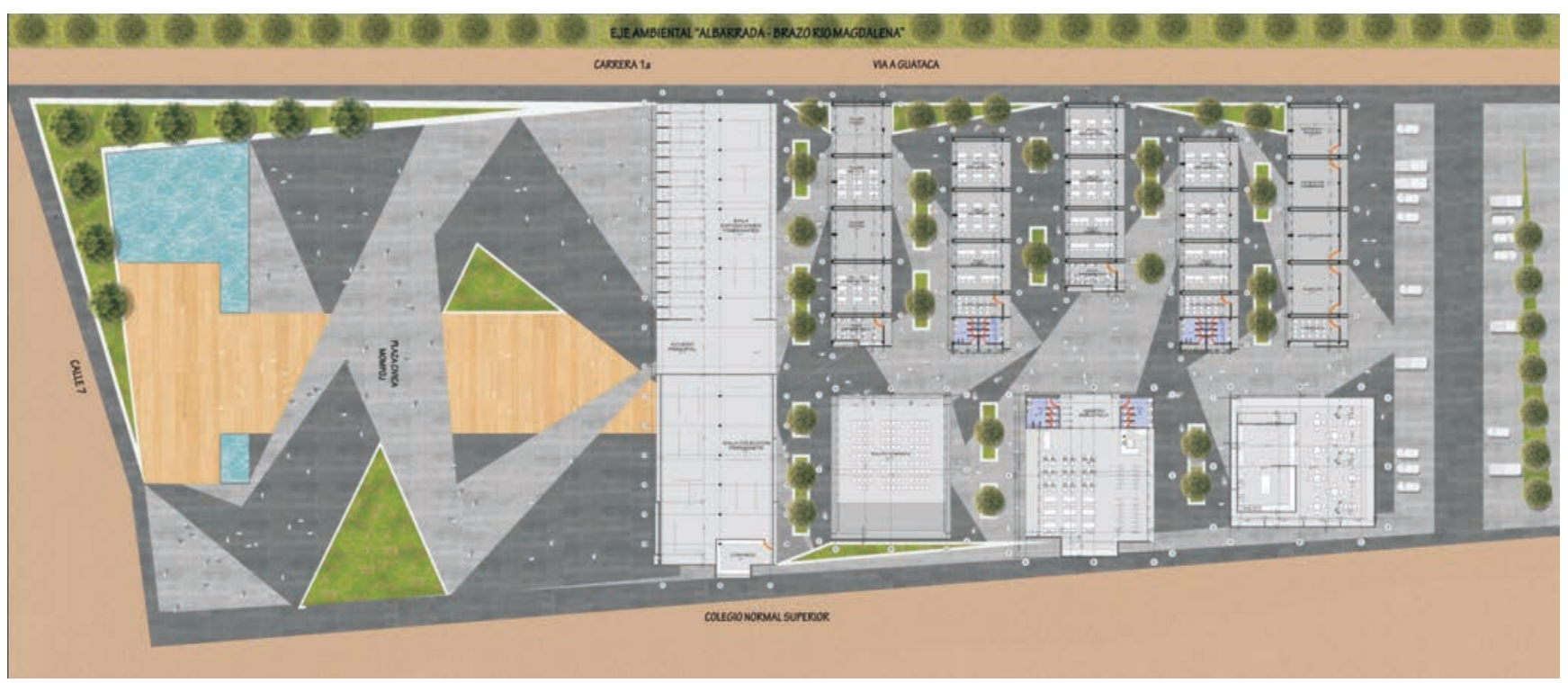




\section{JUSTIFICACIÓN}

El desarrollo del proyecto se torna importante para la región, porque garantiza la educación en relación con la vocación educativa, cultural y turística de la ciudad, incentiva la participación integral de los habitantes y la protección y conservación eficaz para revalorizar el patrimonio cultural y natural del entorno. El proyecto está dirigido a los niños y jóvenes momposinos y pretende generar oportunidades de capacitación y garantizar la educación en diversas ramas del arte y la artesanía. Por lo anterior, el proyecto es útil en tanto quiere promover la calidad educativa a través del desarrollo de aulas y espacios en los cuales sea posible impartir programas dirigidos principalmente a la población infantil y juvenil; así mismo, se diseñaron espacios en los cuales se pudiera ofrecer capacitación a docentes y personas vinculadas laboralmente con el municipio (talleres y conferencias, entre otras), con el propósito de mejorar la formación, capacitar y actualizar en temas relacionados con las artes y la gestión cultural.

Este proyecto educativo se caracterizará por unos ámbitos de formación en las artes plásticas y visuales, literatura, artes escénicas, nuevas tecnologías y pedagogía de las artes. El proyecto, de vocación educativa, estuvo dirigido a la comunidad en general, aunque se diseñaron escenarios exclusivamente destinados a la formación de la población infantil y juvenil, al tiempo que se contempló en la propuesta, la adecuación del espacio para el uso de personas con discapacidad.

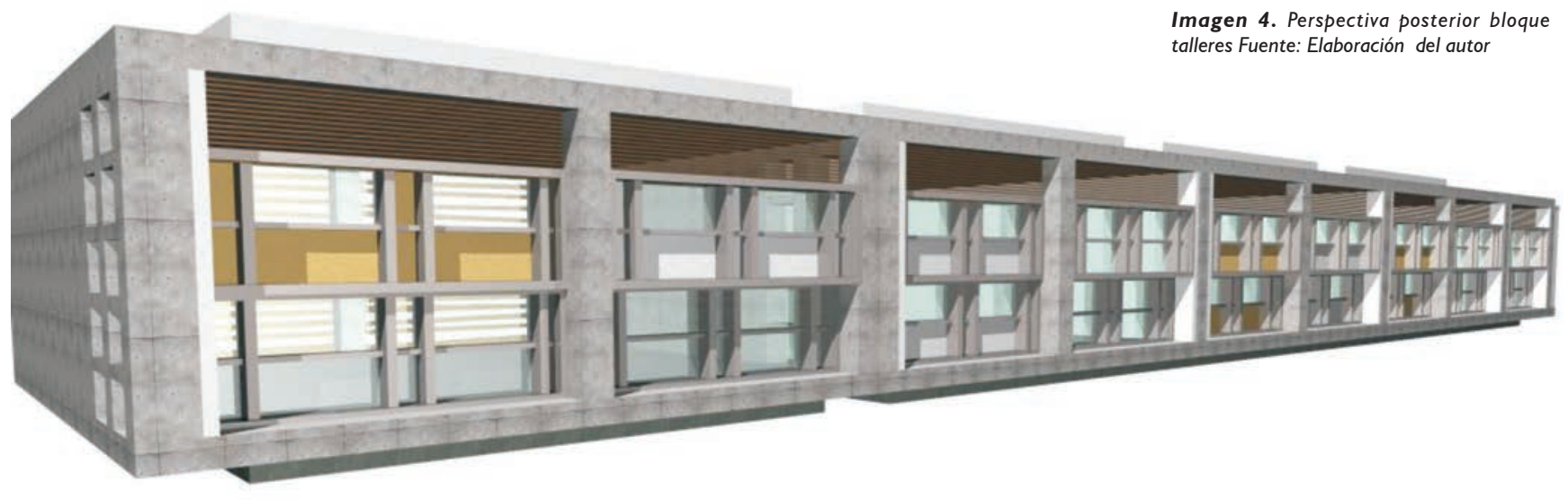

\section{OBJETIVOS}

General: Diseñar un Centro de Bellas Artes en Mompox, con el propósito de generar espacios en los cuales puedan desarrollarse programas educativos a nivel artístico, para difundir la creación y la expresión de prácticas culturales y artísticas en relación con las nuevas tecnologías y promocionar el arte como medio integrador de la comunidad con su entorno.

\section{Específicos}

- Se contempla, en el desarrollo del proyecto, la creación de espacios en los cuales se eduque a la población en temas relacionados con las artes y la cultura (aulas, talleres, sala de exposiciones, biblioteca) 
Imagen 5. Salón comunal. Fuente: Elaboración del autor
- Motivar la participación de la comunidad momposina en relación con el proyecto, para ello se pensó en la proyección de escenarios cubiertos al aire libre.

- Mejorar las condiciones socio-económicas de los participantes, por medio de la promoción de los productos realizados en los talleres de formación (artesanías, cuadros y esculturas, entre otros), se diseña para ello una tienda en la cual los estudiantes puedan exhibir el resultado de sus trabajos.

- Promover el desarrollo de las artes a través de muestras y encuentros de artistas, para lo cual fue necesario diseñar salones de exposición y por último, promover el turismo en el municipio por medio de muestras artísticas (eventos, festividades, oficios, técnicas tradicionales, gastronomía)

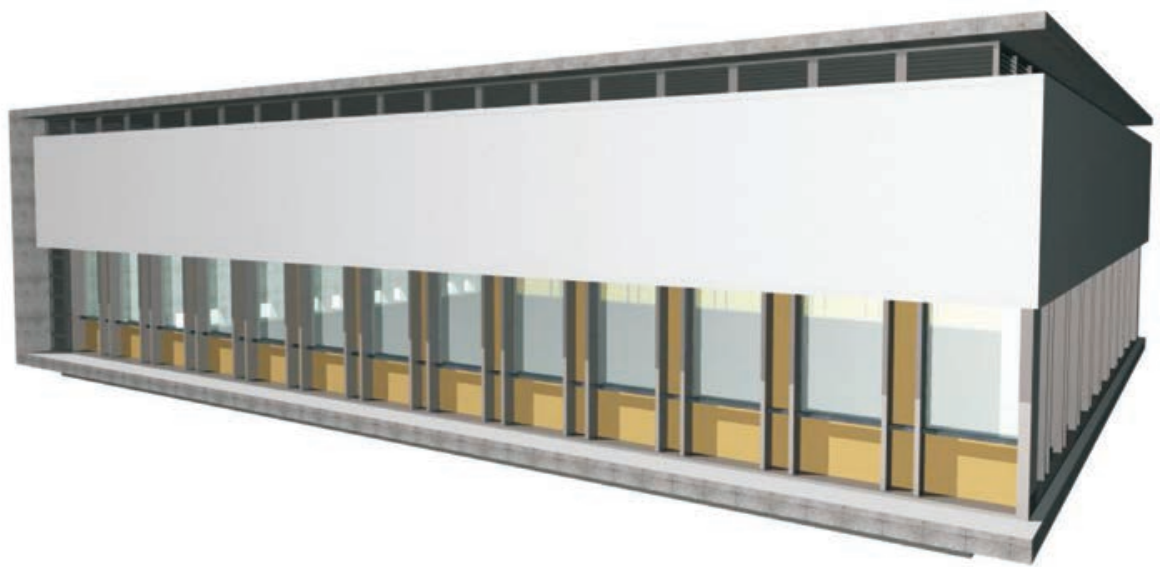

Centro Cultural. El concepto de centro cultural hace referencia a un establecimiento o elemento arquitectónico, de carácter público y continuo, proyectado para servir como espacio de producción, formación académica, conservación, interpretación y difusión de los bienes culturales y artísticos de una comunidad, involucrando de manera activa a los habitantes y proporcionando el espacio requerido para el desarrollo de las personas en esta área.

Este elemento tiene, a su vez, como función el estudio, la valoración y exposición de una variada gama de elementos tanto artísticos como históricos, tecnológicos y científicos, con el fin de servir como medios de educación y deleite de la comunidad. De esta manera se puede ofrecer en el diseño, una serie de espacios en los cuales los artistas puedan manifestar sus creaciones para fortalecer el vínculo entre la ciudadanía y la cultura.

\section{LOCALIZACIÓN DEL SECTOR DE IMPLANTACIÓN}

El sector en el cual se pensó implantar el proyecto en el municipio de Mompox, Bolívar, se encuentra ubicado entre la Carrera I vía a Guataca y la Calle 7, en el barrio Santa Cruz. El lote se encuentra por fuera del sector delimitado como centro histórico y cultural de Mompox y hace parte del lote del Colegio Normal Superior. Está definido por el Plan Básico de Ordenamiento Territorial del municipio de Mompox, con un uso dotacional el cual se define a continuación:

“aquellos usos urbanos cuya función es permitir la realización de actividades para la prestación de diferentes servicios tendientes a proveer a los ciudadanos de los equipamientos básicos y complementarios para el desarrollo integral y soporte de sus actividades. Pueden ser de carácter público, privado o mixto”. 
Es importante rescatar que es un sector que tiene como factor urbano importante, la vía de la Carrera I, la cual permite la comunicación con otros municipios y el eje ambiental "Albarrada". Esta vía es catalogada como VI según el Plan Básico de Ordenamiento Territorial de Mompox y presenta un flujo considerable en el transporte tanto público como privado por su conexión con otros municipios; ésta determinante se presenta como una oportunidad potencial para que el objeto arquitectónico pueda ser un elemento accesible y reconocible fácilmente para la ciudadanía. La Calle 7 es catalogada como V3.

El análisis del sector puso en evidencia que el lote se encuentra rodeado en menos de un rango de 3 Kilómetros, por diferentes instituciones culturales como la Biblioteca, el Museo, la Casa de la Cultura, centros educativos, equipamientos religiosos, plazas y parques.

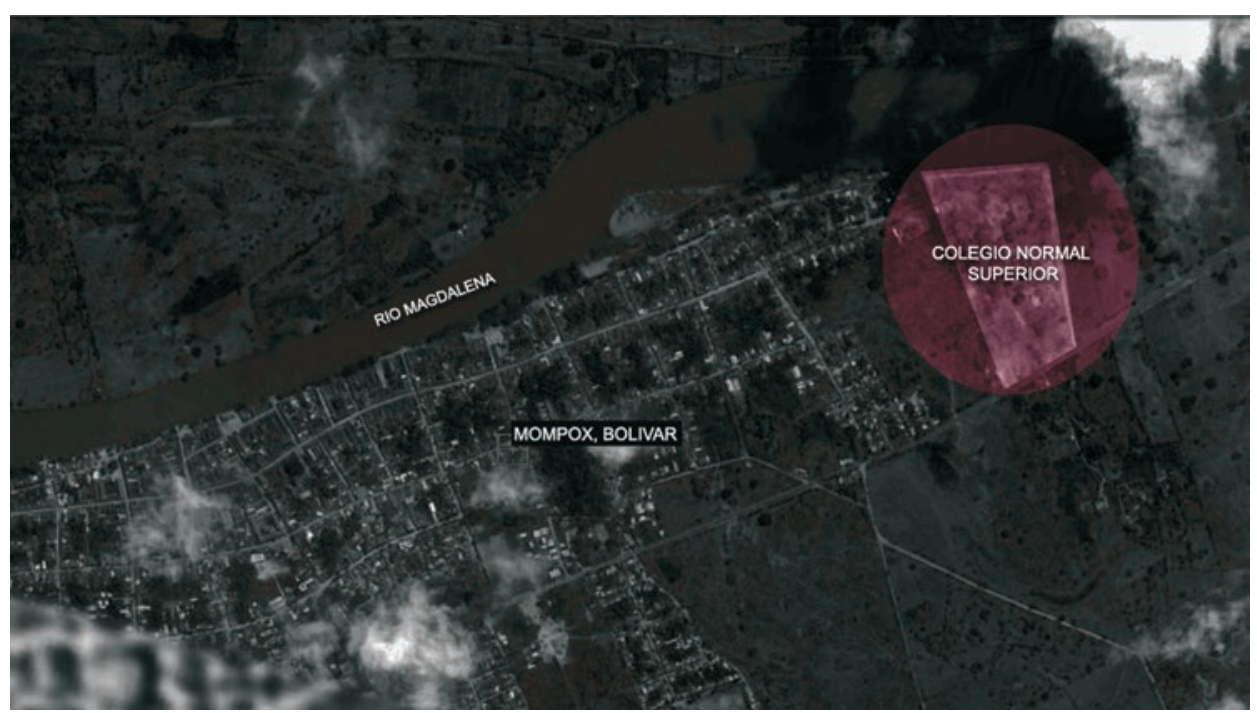

\section{INDICADORES DEL LOTE}

El lote presenta un área neta de $18.557 \mathrm{~m}^{2}$, el área construida es de $5.522 \mathrm{~m}^{2}$. El I.O es de $40 \%$ y el I.C es de I.2; de esta manera, el proyecto cumpliría con lo establecido en el Acuerdo No. 001 de 1994, que en el artículo 63 del subcapítulo 2, establece para una obra nueva o modificación, la altura libre en primer piso de mínimo de 2.80 metros; en tanto que en los pisos adicionales será como mínimo de 2.50 metros; así mismo, en el artículo 66 se indican los índices de ocupación y construcción, en los cuales el área ocupada por la edificación no podrá exceder al $60 \%$ del área total del lote. El índice máximo de construcción será I.2, en los predios con afectación de altura la ocupación máxima será del $70 \%$ y el índice de construcción será de I.4.

\section{DESARROLLO DE LA PROPUESTA URBANA}

En el desarrollo de la propuesta se contemplaron aspectos importantes para la integración de los espacios culturales y artísticos del municipio, con respecto al objeto arquitectónico proyectado, se fomenta en el diseño los oficios propios de Mompox para complementarlos con otras artes, de esta manera se pretende que el equipamiento actúe en el espacio como un nodo educativo, cultural y urbano. En relación con lo urbano, el proyecto se enlaza con el resto del municipio a través de la Plaza de Mompox, tratamiento por medio del cual se promueve un desarrollo posterior que contempla la integración con los diferentes centros educativos y urbanos del municipio.
Plano 2. Ubicación del lote Fuente: Fotografía satelital de Google Earth, intervenida por el autor. 
El proyecto promueve la recuperación integral, tanto urbana como social del municipio, ya que por su función, el equipamiento contempla espacios en los cuales es posible prestar un servicio a la comunidad en lo que respecta a educación y promoción de las artes y la cultura, al tiempo que ofrece la posibilidad de desarrollar eventos diversos; en síntesis, se buscó a través del diseño la recuperación física y espacial del sector de forma tal que se revitalice como un área activa, primordial para la ciudad, donde se promueva el desarrollo de una manera sostenible, un tema de interés no sólo para la administración municipal, sino también para los habitantes de Mompox.

Se generaron conexiones a través de las vías: Carrera I ${ }^{a}$ y Calle 7, con el fin de lograr un sistema de movilidad penetrable al Centro de Artes y Oficios de Mompox y éste, a su vez, en relación con su entorno. Este tipo de conexión pretende convertirse en una red de conexión educativa, cultural y urbana, que fortalece el uso del objeto arquitectónico, como espacio complementario de todas las actividades que se desarrollan en el municipio.

Con la implantación del proyecto se plantea consolidar el eje ambiental del centro histórico "La Albarrada", con el fin de dar continuidad al eje ambiental, fortalecer la movilidad peatonal y la conexión entre el centro histórico y el objeto arquitectónico, razón por la cual, el proyecto articula la estructura urbana del municipio complementándolo con un espacio público denominado "Plaza de Mompox" que incentiva el mejoramiento y conciliación de los espacios públicos allí establecidos.

El ejercicio académico pretendió, por tanto, ofrecer un espacio para la inclusión social, el encuentro, la convivencia y el acceso a la educación, por medio de la promoción de espacios adecuados para el desarrollo de las artes y oficios.

\section{CONCLUSIONES}

- El tratamiento del objeto arquitectónico ha sido propuesto como nodo cultural y educativo, en la medida en que soluciona problemas del espacio público cultural y el sector de la educación en las artes y oficios.

- La Arquitectura y el Arte fueron las directrices para desarrollar un objeto arquitectónico en el cual se promoviera la igualdad para la comunidad, sin restricciones. De allí que el objetivo consistiera en plantear diferentes sistemas formales con el fin de responder a las necesidades de la comunidad y lograr, de esta manera, que los habitantes se identificaran con el proyecto.

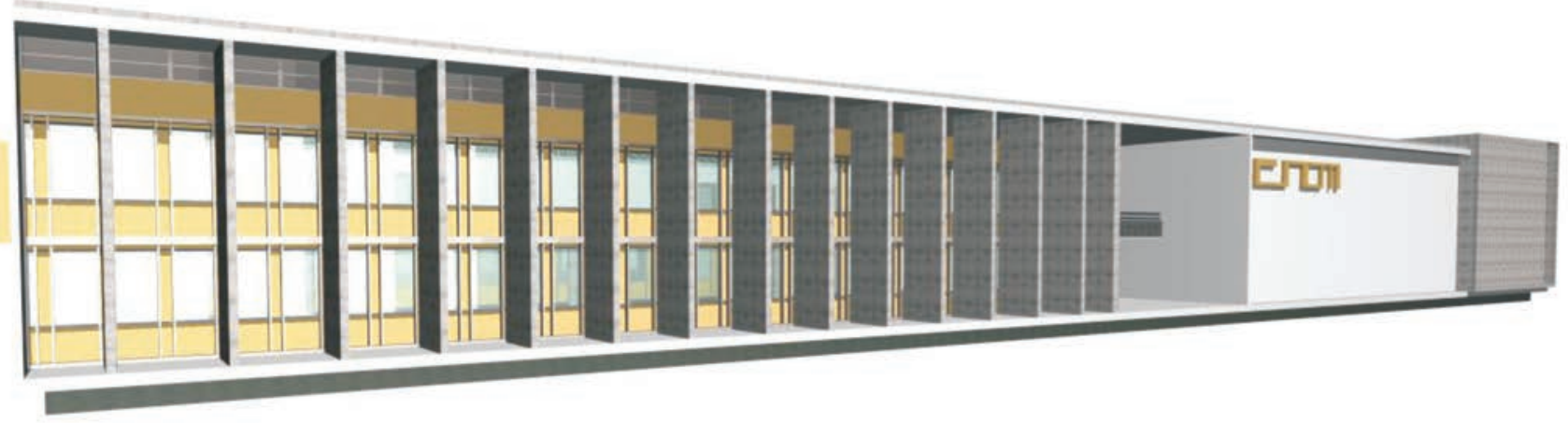

Imagen 6. Perspectiva frontal Fuente: Elaboración del autor 
- Con la propuesta de diseño se pretende fortalecer y mejorar las actividades culturales y educativas existentes en Mompox y prestar a la comunidad un servicio básico de actividades, además, fomentar el aprendizaje y la enseñanza entre los usuarios potenciales.

- El proyecto planteado como nodo educativo, permitió ordenar, establecer y estructurar patrones de aprendizaje, ya que en Mompox predomina el desarrollo de técnicas ancestrales como la filigrana, actividad que encuentra cabida en el diseño, pues en él se generaron espacios en los cuales es posible cultivar este medio de expresión, tan importante para la comunidad momposina.

- Se trata de un espacio que genera identidad y arraigo por el entorno socio cultural e histórico de la zona, un equipamiento destinado para enriquecer la imaginación, la motricidad y las sensaciones, a través del aprendizaje y la promoción de la cultura, para despertar el interés por las cosas nuevas, fortaleciendo la capacidad creativa de sus habitantes.

\section{BIBLIOGRAFÍA}

CORRADINe ANGULO, Alberto. Santa Cruz de Mompox, Estudio Morfológico y Reglamentario. Ministerio de Desarrollo Económico, Corporación Nacional de Turismo, Bogotá, Op Gráficas LTDA, 1981.

SALZEDO DEL VILLAR, Pedro. Apuntaciones Historiales de Mompox, Cartagena, Tipografía Democracia, 1938.

ZABALA CORREDOR, Sandra Karime [Consultora] Plan Especial de Manejo y Protección del Centro Histórico de Santa Cruz de Mompox, Convenio No. 1403 de 2007. Agencia Española de Cooperación para el desarrollo, Ministerio de Cultura, Alcaldía de Mompox, Gobernación de Bolívar. Licitación Pública No. 004 de 2007. Contrato 002 de 2007, Alcaldía Mompox. 